\title{
The Role of Software-Defined Networking in Cellular Networks ${ }^{\dagger}$
}

\author{
Pablo Fondo-Ferreiro *(D) and Felipe Gil-Castiñeira \\ atlanTTic Research Center, University of Vigo, 36310 Vigo, Pontevedra, Spain \\ * Correspondence: pfondo@gti.uvigo.es; Tel.: +34-986-818-684 \\ + Presented at the XoveTIC Conference, A Coruña, Spain, 5-6 September 2019. \\ Published: 31 July 2019
}

\begin{abstract}
In this paper, we discuss how SDN can contribute to enhance future cellular networks. We first present SDN and describe its characteristics. Then, we explore how SDN can be used to improve current cellular networks, analyzing the advantages and disadvantages, while highlighting some use cases. Finally, we conclude this work exposing some challenges that still require further research to take full advantage of SDN in cellular networks.
\end{abstract}

Keywords: cellular networks; mobile networks; mobile core network architecture; software-defined networking; SDN

\section{Introduction}

Since the advent of mobile phones, cellular networks became one of the most relevant communication systems. Moreover, cellular networks are embracing more applications, supporting a wide range of connectivity typologies with different types of requirements, such as those coming from Internet of Things (IoT), Industry 4.0 or connected cars. The growing demand for ubiquitous connectivity is leading operators to enhance their current networks in terms of scalability, adaptability and performance. Yet many efforts are being focused on the evolution of the radio access network (RAN) by exploring new frequencies, antennas and modulation techniques, the core network (CN) also needs to be evolved, in order to properly manage the increased amount of traffic.

New network architectures such as Network Slicing and Multi-Access Edge Computing (MEC) have been identified to play a key role in satisfying the diverse requirements brought by the new use cases. However, in order to implement such architectures it is essential to have flexible and programmable networks. Two networking paradigms, namely Software-Defined Networking (SDN) and Network Function Virtualization (NFV), are key enablers to build these networks.

\section{Software-Defined Networking}

Software-Defined Networking (SDN) [1] is an architecture that decouples the control plane from the data plane in networking devices. Besides, the control plane is centralized in a logical entity named the SDN controller. The network is then programmable through software applications running on top of the SDN controller.

This paradigm enables flexibility by breaking the vertical integration in traditional IP networks where control and data planes are combined inside the same networking device. In SDN, controllers follow policies specified by SDN applications to decide where packets should be forwarded (i.e., the control plane), whereas SDN enabled devices, such as switches or routers, execute the forwarding actions (i.e., data plane), as shown in Figure 1.

In SDN networks forwarding decisions are now flow-based, where a flow is defined as an artificial logical equivalent to a call or connection [2]. Thus, SDN networks can define complex sets of rules 
(which can be modified dynamically at the controller) that match different packet header fields to establish a set of actions that will handle the network traffic.

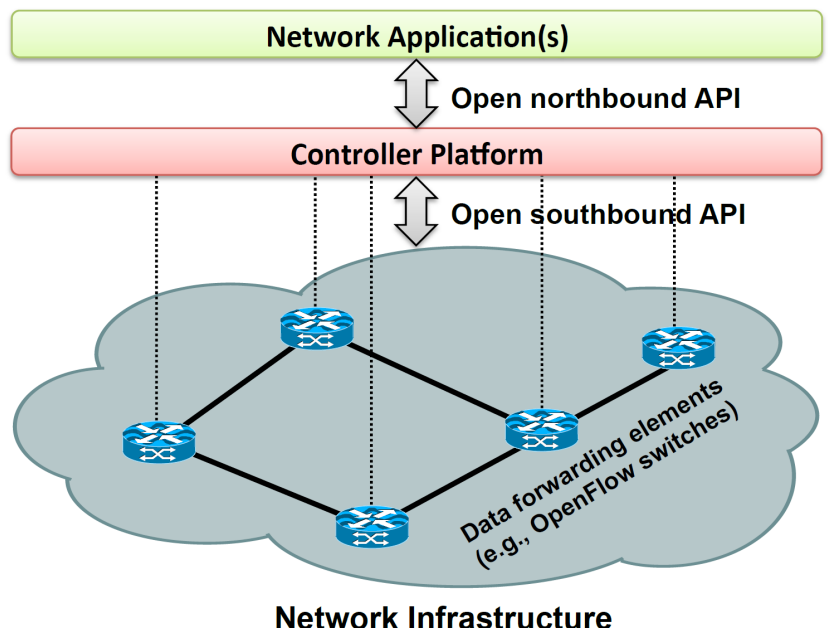

Figure 1. SDN architecture. Retrieved from [1].

\section{SDN in Mobile Cellular Networks}

The SDN technology enables flexible networks that will be the key to implement the functionalities that are expected to be provided by the Core Network $(\mathrm{CN})$ of the future cellular networks. In this section, we present the major directions for introducing SDN in the core of the mobile network architecture. There are three main ways to re-architect the Evolved Packet Core (EPC) of the mobile cellular networks according to [3]: the first one consists in virtualizing the EPC using NFV techniques, the second one separates the control and user planes in the EPC using SDN, and the third one consists in a "clean-slate" fully SDN based architecture.

The first approach consists in migrating conventional EPC entities to Virtual Machines (VMs) or containers. This way, the protocols and interfaces standardized by the 3GPP are maintained. This approach introduces benefits such as cost reduction, flexibility, and simplicity in the deployment, since it does not require any major change, and it is interoperable with current deployments. However, this approach presents several limitations: difficult management, orchestration, scaling and resilience problems. This approach was, for example, discussed in [4].

The second group of proposals put their focus on the data plane entities of the core network, that is, the Serving Gateway (SGW) and Packet Data Network Gateway (PGW) in 4G cellular networks. These entities are divided into control plane (SGW-C and PGW-C) and data plane (SGW-U and PGW-U), following the decoupling proposed by the SDN paradigm. Some proposals maintain the data plane entities as hardware elements (for performance purposes) while others virtualize them. All of these proposals employ an SDN controller for configuring the forwarding rules of the data plane devices. The main advantages of these architectures are the flexibility introduced by SDN and also the independent scalability of control and data planes. On the other hand, the introduction of the SDN controller slightly increases the latency in the control plane of the network. Besides, keeping 3GPP protocols in the architecture maintains the overhead that they introduce. This type of architecture was initially proposed in [5].

The third approach proposes a clean-slate SDN based revolutionary architecture, not influenced by the entities, interfaces and protocols standardized by the 3GPP. Most of these proposals depict the following simplified architecture: a control plane implemented as SDN applications running on top of an SDN controller, and a data plane composed of SDN switches and middleboxes. The main benefits are the improved flexibility and reduced overhead due to the elimination of the specific protocols such as GTP. However, despite the simplicity of the architecture, the complexity of the control plane and 
QoS enforcing policies imposes challenges in the implementation. In addition, it should also be noted that this approach is not interoperable with current deployments. Further information can be found in [6].

Furthermore, we can also consider using an SDN network as the transportation network interconnecting the elements of the EPC. The main advantage of this simple approach is that it can be quickly deployed without the need to modify any entity, while being interoperable with existing deployments. Besides, it introduces some flexibility in the traffic management, facilitating the balancing of traffic among different EPC entities. On the contrary, the main drawback is the remaining overhead due to the operation of conventional EPC entities.

Finally, Network Slicing is a practical use case that can clearly take advantage of the application of the SDN paradigm to the core of the cellular networks. SDN can be used in the transport network to redirect traffic to the appropriate Network Slice, just by programming the SDN controller to manage traffic flows according to their slice.

\section{Conclusions}

Current and future cellular networks can benefit from the application of some recent networking paradigms such as SDN and NFV. For example, MEC and Network Slicing paradigms will require flexible CNs, as the ones that can be built with SDN. Furthermore, we have summarized the proposals of different authors who have considered using SDN to reinvent the cellular network architecture by taking advantage of the flexibility and programmability provided by SDN. As future work, we will continue exploring how new networking paradigms can be used to optimize and improve cellular networks.

Author Contributions: Conceptualization, F.G.-C.; investigation, P.F.-F.; writing-original draft preparation, P.F.-F.; writing—review and editing, F.G.-C.; supervision, F.G.-C.; funding acquisition, P.F.-F. and F.G.-C.

Funding: This work has been supported by "la Caixa" Foundation (ID 100010434) fellowship code LCF/BQ/ES18/11670020.

Conflicts of Interest: The authors declare no conflict of interest. The founding sponsors had no role in the design of the study; in the collection, analyses, or interpretation of data; in the writing of the manuscript, or in the decision to publish the results.

\section{References}

1. Kreutz, D.; Ramos, F.M.; Verissimo, P.; Rothenberg, C.E.; Azodolmolky, S.; Uhlig, S. Software-defined networking: A comprehensive survey. Proc. IEEE 2015, 103, 14-76.

2. Carpenter, B.E.; Deering, D.S.E.; Rajahalme, J.; Conta, A. IPv6 Flow Label Specification; RFC 3697; Internet Engineering Task Force (IETF): Fremont, CA, USA, 2014, doi:10.17487/RFC3697

3. Nguyen, V.G.; Brunstrom, A.; Grinnemo, K.J.; Taheri, J. SDN/NFV-based mobile packet core network architectures: A survey. IEEE Commun. Surv. Tutor. 2017, 19, 1567-1602.

4. Yousaf, F.Z.; Lessmann, J.; Loureiro, P.; Schmid, S. SoftEPC—Dynamic instantiation of mobile core network entities for efficient resource utilization. In Proceedings of the 2013 IEEE International Conference on Communications (ICC), Budapest, Hungary, 9-13 June 2013; pp. 3602-3606.

5. Kempf, J.; Johansson, B.; Pettersson, S.; Lüning, H.; Nilsson, T. Moving the mobile evolved packet core to the cloud. In Proceedings of the 2012 IEEE 8th International Conference on Wireless and Mobile Computing, Networking and Communications (WiMob), Barcelona, Spain, 8-10 October 2012; pp. 784-791.

6. Jin, X.; Li, L.E.; Vanbever, L.; Rexford, J. Softcell: Scalable and flexible cellular core network architecture. In Proceedings of the Ninth ACM Conference on Emerging Networking Experiments and Technologies, Santa Barbara, CA, USA, 9-12 December 2013; pp. 163-174.

(C) 2019 by the authors. Licensee MDPI, Basel, Switzerland. This article is an open access article distributed under the terms and conditions of the Creative Commons Attribution (CC BY) license (http:/ / creativecommons.org/licenses/by/4.0/). 